\section{Sedentary Behavior and Diabetes Risk Among Women Over the Age of 65 Years: The OPACH Study}

Diabetes Care 2021;44:563-570 | https://doi.org/10.2337/dc20-0709

\section{OBJECTIVE}

To evaluate whether sedentary time (ST) and/or sedentary behavior patterns are related to incident diabetes in the U.S.'s oldest age-groups.

\section{RESEARCH DESIGN AND METHODS}

Women without physician-diagnosed diabetes $(n=4,839$, mean \pm SD age $=79 \pm 7$ years) wore accelerometers for $\geq 4$ days and were followed up to 6 years for selfreported newly diagnosed diabetes requiring treatment with medications. Hazard ratios (HRs) for incident diabetes were estimated across quartiles of accelerometermeasured ST and mean bout duration with use of Cox proportional hazards models. We conducted isotemporal substitution analyses using Cox regression and tested associations with risk for diabetes after statistically replacing ST with light physical activity (PA) or moderate-to-vigorous PA (MVPA) and after replacing light PA with MVPA.

\section{RESULTS}

During 20,949 person-years, 342 diabetes cases were identified. Women in ST quartile (Q)2, Q3, and Q4 (vs. Q1) had incident diabetes HR 1.20 (95\% Cl 0.871.65), $1.33(0.97-1.82)$, and $1.21(0.86-1.70) ; P_{\text {trend }}=0.04$. Respective HRs following additional adjustment for BMI and MVPA were 1.04 (95\% Cl 0.741.47), $1.04(0.72-1.50)$, and $0.85(0.56-1.29) ; P_{\text {trend }}=0.90$. Fully adjusted isotemporal substitution results indicated that each $30 \mathrm{~min}$ of ST replaced with MVPA (but not light PA) was associated with 15\% lower risk for diabetes (HR 0.85 [95\% $\mathrm{Cl} 0.75-0.96] ; P=0.01)$; the HR for replacing 30 min of light PA with MVPA was 0.85 (95\% $\mathrm{Cl} 0.73-0.98) ; P=0.03$. Mean bout duration was not associated with incident diabetes.

\section{CONCLUSIONS}

Statistically replacing ST or light PA with MVPA was associated with lower diabetes risk in older women. While reducing ST is important for several health outcomes, results indicate that to reduce diabetes risk among older adults, the primary public health focus should be on increasing MVPA.

Type 2 diabetes is epidemic in the U.S., affecting $13.0 \%$ of those over the age of 17 years and $28.6 \%$ of those over age 64 years (1). Among adults with diabetes, older adults are at the highest risk for complications such as vascular disease, renal impairment, and severe or fatal hypoglycemia (2-4). Furthermore, diabetes accelerates the decline of physical functioning (5), impacting key aspects of healthy aging such as independence and overall quality of life.
John Bellettiere, ${ }^{1,2}$ Michael J. LaMonte, Genevieve N. Healy, ${ }^{4,5,6}$ Sandy Liles, ${ }^{1,2}$ Kelly R. Evenson, ${ }^{7}$ Chongzhi Di, ${ }^{8}$ Jacqueline Kerr, ${ }^{1}$ I-Min Lee, ${ }^{9}$ Eileen Rillamas-Sun, ${ }^{8}$ David Buchner, ${ }^{10}$ Melbourne F. Hovell, ${ }^{2,11}$ and Andrea Z. LaCroix ${ }^{1}$

${ }^{1}$ Department of Family Medicine and Public Health, University of California, San Diego, La Jolla, CA

${ }^{2}$ Center for Behavioral Epidemiology and ComCenter for Behavioral Epidemiology and Com-
munity Health, Graduate School of Public Health, San Diego State University, San Diego, CA

${ }^{3}$ Department of Epidemiology and Environmental Health, School of Public Health and Health Professions, State University of New York at Buffalo, Buffalo, NY

${ }^{4}$ School of Public Health, University of Queensland, Queensland, Australia

${ }^{5}$ Baker IDI Heart and Diabetes Institute, Melbourne, Victoria, Australia ${ }^{6}$ School of Physiotherapy, Curtin University, Perth,
Western Australia, Australia

${ }^{7}$ Department of Epidemiology, Gillings School of Global Public Health, University of North Carolina at Chapel Hill, Chapel Hill, NC

${ }^{8}$ Division of Public Health Sciences, Fred Hutchinson Cancer Research Center, Seattle, WA

${ }^{9}$ Division of Preventive Medicine, Brigham and Women's Hospital, Harvard Medical School, Boston, $M A$

${ }^{10}$ Department of Kinesiology and Community Health, University of Illinois at Urbana-Champaign, Champaign, IL

${ }^{11}$ Division of Health Promotion and Behavioral Science, Graduate School of Public Health, San Diego State University, San Diego, CA

Corresponding author: John Bellettiere, jbellettiere@ ucsd.edu

Received 1 April 2020 and accepted 30 October 2020

This article contains supplementary material online at https://doi.org/10.2337/figshare.13224992. 
Each year, $0.9 \%$ of older adults are diagnosed with diabetes for the first time (1). As many as 9 out of 10 new cases can be attributed to modifiable lifestyle factors such as physical inactivity, poor diet, smoking, alcohol use, and BMI (obesity) (6). Several review articles conclude that sedentary behavior (i.e., waking behavior requiring low energy expenditure and occurring in a sitting, reclining, or lying posture) (7) is another important contributor to the development of diabetes (e.g., 8). However, nearly all the studies reviewed relied on self-reported measures of sedentary behavior, which are inaccurate (9), and few focused on older adults, for whom sedentary time (ST) is higher than at younger ages and in whom these behaviors are especially challenging to measure with questionnaires.

Recent improvements in technology and cost-efficiency have permitted use of accelerometers in population studies of sedentary behavior and health outcomes. Several studies have examined associations between accelerometer-measured sedentary behavior and risk factors related to diabetes (10). Just three studies (11-13) examined the cross-sectional association of ST with diabetes as a clinical end point, and all found higher odds of diabetes with higher ST. The only prospective study to date examining ST and diabetes incidence did not find a significant association among 1,718 adults mean \pm SD age $=45 \pm 3$ years with 5 years of follow-up (11). The paucity of highquality evidence relating sedentary behavior to incident diabetes, especially among older adults, has led to consensus that evidence from prospective studies that measure sedentary behavior using accelerometers is a high research priority (14).

To address this research priority, we examined associations of accelerometermeasured ST and ST patterns with incident diabetes in 4,834 older women. Our hypotheses were that women with higher cumulative ST and/or a pattern of frequent prolonged sedentary bouts at baseline would have higher risk for diabetes. A secondary aim of the study was to examine whether statistically replacing ST with light-intensity physical activity (PA) or moderate-to-vigorous PA (MVPA) was associated with diabetes risk. Findings will address key evidence gaps regarding the role of sedentary behavior, and sedentary behavior patterns, in the development of late-life diabetes.

\section{RESEARCH DESIGN AND METHODS}

\section{Study Participants}

As part of the Women's Health Initiative (WHI) 2010-2020 Extension Study and the ancillary Long Life Study conducted from 2012 to 2013, 7,058 ambulatory community-living women aged 63 years and older were enrolled in the Objective Physical Activity and Cardiovascular Disease Health in Older Women (OPACH) Study. Detailed methods have previously been published (15). Briefly, at OPACH Study baseline (March 2012-April 2014), women were asked to wear ActiGraph GT3X + accelerometers at their hip secured to an elastic belt around their waist $24 \mathrm{~h} /$ day for 7 days (except when showering or swimming) and to concurrently record in-bed and out-of-bed times using sleep logs. Women were subsequently followed annually for morbidity and mortality as part of annual WHI follow-up. Of the 6,489 (91.9\%) women enrolled in the OPACH Study who returned accelerometers, 6,133 were included in the current study because they met the commonly used wear criteria of having at least 4 days, each with $\geq 10$ h of awake wear time (16). After exclusion of 12 women with missing diabetes follow-up data and 1,282 women with physician-diagnosed diabetes at $\mathrm{OPACH}$ Study baseline, the analytic sample for this study included 4,839 women who were at risk for developing diabetes during follow-up.

\section{Identification of Diabetes}

At WHI baseline, between 1993 and 1998, participants were asked, "Did a doctor ever say that you had sugar diabetes or high blood sugar when you were not pregnant?" Then, at regular intervals (at least annually) during follow-up, standardized medical history updates were mailed to participants that included questions about new physician-diagnosed diabetes requiring insulin or oral hypoglycemic medication. Incident diabetes cases were defined as any participant reporting physician-diagnosed diabetes treated with insulin or oral medication after OPACH Study baseline through 31 March 2018. With the same case definition, a separate study of 715 WHI participants showed that reported incident diabetes was concordant with expert medical record review in $82 \%$ of reported confirmed cases; reports of being without diabetes were concordant in $95 \%$ of women confirmed as not having diabetes (17).

\section{ST, ST Patterns, and PA}

Accelerometer data measured at $30 \mathrm{~Hz}$ were converted to 1-min epochs with use of the low-frequency extension filter and to 15-s epochs with the normal filter in ActiLife, version 6 . With the Choi algorithm we identified data collected while devices were unworn; periods while participants were in bed were identified with use of recorded times from sleep diaries. When at least one in-bed or out-of-bed time was missing, each woman's average time was used if available; otherwise, the overall mean in-bed (10:45 P.M.) and outof-bed (7:22 A.M.) times were used.

The accelerometer cut points for classifying sedentary behavior, light PA, and MVPA-determined in a laboratorybased calibration study of $200 \mathrm{WHI}$ participants aged 60-91 years-were 0-18, 19-518, and $>518$ vector magnitude counts/15 s, respectively (18). How ST is accrued in long and short uninterrupted periods (sedentary bouts) describes one's ST patterns. Using the acceleration cut points described above to measure ST patterns was problematic because vector magnitude acceleration values within 15-s epochs were overly sensitive to breaks in ST (i.e., in our sample, on average there were $>300$ breaks in ST per day). Thus, to classify breaks, we measured ST using a common ActiGraph data-processing protocol for older adults (100 counts/min applied to the vertical axis using the low-frequency extension filter) (16) and then identified bouts of consecutive sedentary minutes that were below this threshold, with no minimum bout duration required. With use of this data processing protocol that we have successfully implemented in previous studies (e.g., 13), the 86 average breaks/day observed in the OPACH Study were similar to those previously reported in another separate sample of older women (19). We then computed our measure of ST patterns as the arithmetic mean sedentary bout duration, using all adherent days from each participant. High mean bout durations indicated prolonged ST patterns; low mean bout durations indicated frequently interrupted patterns.

\section{Covariates}

Age, race/ethnicity, education level, and family history of diabetes were measured by questionnaire at WHI baseline. Family history of diabetes was assessed with the 
question, "Did your mother or father, or full-blooded sisters, full-blooded brothers, daughters, or sons ever have sugar diabetes or high blood sugar that first appeared as an adult?" Self-reported health status, physical functioning (from the RAND 36-Item Short-Form Survey [SF-36]) (20), alcohol consumption, and current smoking status (smoker, nonsmoker [missing values were classified as nonsmokers]) were measured by questionnaire at OPACH Study baseline. Near OPACH Study baseline, trained research assistants conducted inperson visits as part of the Long Life Study; measured height and weight, which was used to compute BMI; and drew blood for biomarker assays including fasting glucose. Multimorbidity was measured as the number of chronic health conditions (cardiovascular disease, cancer, cognitive impairment, depression, osteoarthritis, history of frequent falls, chronic obstructive pulmonary disease, hypertension, cerebrovascular disease) at or before OPACH Study baseline.

\section{Statistical Analysis}

To account for differences in accelerometer wear time while subjects were awake, we adjusted ST for awake wear time using the residuals method. Then, ST, mean bout duration, and covariates were summarized by quartile of ST.

Cox proportional hazards models estimated hazard ratios (HRs) of incident diabetes in relation to ST and mean bout duration, in separate models. Time to event was computed as the number of days from OPACH Study baseline to the date of the returned questionnaire on which diabetes diagnosis was reported, death, or the last medical update received. HRs were estimated for quartiles of each sedentary behavior-related exposure variable. Four models were fit for each sedentary behavior-related exposure variable as follows: model 1 , adjustment for age and race/ethnicity; model 2, model 1 adjustments plus potential confounders (education, family history of diabetes, self-rated health, physical functioning, alcohol consumption, and smoking status); model 3a further controlled for BMI, which can be considered to be on the causal pathway between sedentary behavior and diabetes and was separately tested as an effect modifier; and model $3 \mathrm{~b}$ included model 2 covariates and MVPA, which has previously been conceptualized as a confounder, a mediator, and a competing behavior (21-23). We also report results for model 3c, which includes model 2 covariates plus BMI and MVPA. Tests for linear trend used Cox regression models that included the continuous form of each sedentary behaviorrelated exposure variable. Adherence to Cox proportional hazards assumptions was confirmed by tests based on Schoenfeld residuals and review of plots of the scaled residuals over time. To account for the interdependency of ST and MVPA (24), we fit isotemporal substitution models using model $3 a$ to estimate how statistically replacing ST with equivalent time spent in light-intensity PA or MVPA was associated with incident diabetes. For a complete isotemporal analysis, we also fit a model for statistically replacing light PA with MVPA.

Tests of effect modification were conducted by inclusion in model 2 of a multiplicative interaction term between the exposure and each potential effect modifier that was a priori selected based on our previous work (13): women $<80$ and $\geq 80$ years old, $<30$ and $\geq 30 \mathrm{~kg} /$ $\mathrm{m}^{2},<75$ and $\geq 75$ on the SF-36 (median split), $<43$ and $\geq 43$ min per day of MVPA (median split), and with or without family history of diabetes and according to race/ ethnicity (White, Black, or Hispanic). For visualization of differential associations by subgroups, models were repeated, stratified by each potential modifier. Tests were also performed with use of model 3c.

\section{Sensitivity Analyses}

Subclinical diabetes and its complications could cause increased ST, leading to associations with new-onset diabetes that could reflect reverse causation. Two approaches were used to address this: first, we removed from our analytic sample women reporting newly diagnosed diabetes within the first 6 months after OPACH Study baseline and repeated model 2 and isotemporal substitution models. Second, from a subsample of 3,832 women who received phlebotomy as part of the Long Life Study, we removed 147 women with fasting glucose $\geq 126 \mathrm{mg} / \mathrm{dL}$ and repeated model 2 and isotemporal substitution models. We also repeated isotemporal substitution models after additional adjustment for fasting glucose and insulin to test whether associations were present independent of these expected mediators. Finally, we repeated all analyses using a consistent sample from model $3 \mathrm{c}(n=$
4,438 ) to test whether using complete cases analyses influenced changes in estimates.

All statistical tests were two tailed, with $\alpha=0.05$, and conducted using $R$ statistical software ( $R$ Foundation for Statistical Computing, Vienna, Austria).

\section{RESULTS}

Mean \pm SD age for participants was $78.9 \pm$ 6.7 years, and $53.1 \%$ were White, $30.4 \%$ Black, and $16.6 \%$ Hispanic. During a mean follow-up time of $4.3 \pm 1.2$ years, 342 women reported being newly diagnosed with diabetes requiring oral medication or insulin. Health-related characteristics of the cohort are described in Table 1. Generally, women in the fourth quartile (quartile [Q]4) of ST were oldest and in the poorest health as measured by selfrated health and physical functioning. Women in the highest ST quartile also had the lowest levels of MVPA (26.9 \pm 17.4 min per day) and light PA (205.1 \pm $34.8 \mathrm{~min}$ per day) and the most prolonged ST patterns as measured by mean bout duration (10.3 $\pm 3.2 \mathrm{~min})$.

Crude incidence rates for diabetes per 1,000 person-years in ST Q1, Q2, Q3, and Q4 were 13.3, 16.6, 18.1, and 17.6, respectively (Table 2). Following adjustment for model 2 covariates, women with the highest ST (Q4) had 21\% higher relative risk for diabetes (HR 1.21 [95\% $\left.\mathrm{Cl}=0.86-1.70] ; P_{\text {trend }}=0.04\right)$ than women in Q1. Following adjustment for BMI (Q4 vs. Q1 HR $1.14[95 \% \mathrm{Cl}$ 0.79-1.64]; $P_{\text {trend }}=0.12$ ) and, separately, for MVPA (Q4 vs. Q1 HR 0.92 $\left.[95 \% \mathrm{Cl} 0.62-1.36] ; P_{\text {trend }}=0.76\right), \mathrm{HRs}$ were further attenuated and there was no longer a significant linear trend.

The interaction of ST with BMI in model 2 had a $P$ value of 0.08 with stratified analyses indicating that associations (for 1 interquartile range [IQR] higher ST [2 h/ day]) were stronger among those with $\mathrm{BMI}<30 \mathrm{~kg} / \mathrm{m}^{2}$ (HR 1.29 [95\% Cl 1.031.60]; 203 diabetes cases) than women with higher BMI (HR 0.94 [95\% Cl 0.701.26]; 120 diabetes cases) (Supplementary Table 1). The interaction of ST and family history of diabetes did not reach common $(P<0.05)$ or conservative $(P<0.10)$ thresholds for effect modification, though stratified analyses indicated that one IQR higher of ST was associated with an HR of 1.12 (95\% Cl 0.91-1.38) for women without a family history of diabetes and 1.29 (95\% Cl 0.99-1.68) among women with a family history; $P_{\text {interaction }}=0.11$. 
Table 1-Baseline sociodemographic and health-related characteristics by quartile of ST ( $n=4,839$ ): the OPACH Study (2012-2018)

\begin{tabular}{|c|c|c|c|c|c|}
\hline Characteristics & Q1 (low) & Q2 & Q3 & Q4 (high) & $P$ \\
\hline Age, years, mean $\pm S D$ & $76.6 \pm 6.3$ & $78.5 \pm 6.7$ & $79.3 \pm 6.7$ & $81.4 \pm 6.4$ & $<0.001$ \\
\hline Age category, $n(\%)$ & & & & & $<0.001$ \\
\hline $63-69$ years & $180(14.9)$ & $140(11.6)$ & $104(8.6)$ & $63(5.2)$ & \\
\hline 70-79 years & $589(48.7)$ & $482(39.8)$ & $460(38.0)$ & $333(27.5)$ & \\
\hline 80-89 years & $423(35.0)$ & $545(45.0)$ & $581(48.1)$ & $719(59.4)$ & \\
\hline $90+$ years & $18(1.5)$ & $43(3.6)$ & $64(5.3)$ & 95 (7.9) & \\
\hline Race/ethnicity, $n$ (\%) & & & & & $<0.001$ \\
\hline White & $493(40.7)$ & $606(50.1)$ & $660(54.6)$ & 809 (66.9) & \\
\hline Black & $414(34.2)$ & $395(32.6)$ & $372(30.8)$ & $288(23.8)$ & \\
\hline Hispanic/Latina & $303(25.0)$ & $209(17.3)$ & $177(14.6)$ & $113(9.3)$ & \\
\hline Highest education level, $n$ (\%) & & & & & 0.05 \\
\hline High school or less & $252(20.9)$ & $231(19.2)$ & $246(20.6)$ & $222(18.5)$ & \\
\hline Some college & $429(35.5)$ & 433 (35.9) & $465(38.9)$ & $490(40.8)$ & \\
\hline College graduate & $527(43.6)$ & 541 (44.9) & $483(40.5)$ & $489(40.7)$ & \\
\hline \multicolumn{6}{|l|}{ Health behavior/status } \\
\hline Current smoker, $n(\%)$ & $22(1.8)$ & $28(2.3)$ & $32(2.6)$ & $51(4.2)$ & 0.002 \\
\hline Alcohol intake, $n(\%)$ & & & & & $<0.001$ \\
\hline Nondrinker & $356(29.4)$ & $390(32.2)$ & $403(33.3)$ & $399(33.0)$ & \\
\hline$<1$ drink per week & 422 (34.9) & $382(31.6)$ & $308(25.5)$ & $279(23.1)$ & \\
\hline$\geq 1$ drinks per week & $346(28.6)$ & $357(29.5)$ & 394 (32.6) & $429(35.5)$ & \\
\hline Unknown alcohol intake & 86 (7.1) & $81(6.7)$ & $104(8.6)$ & $103(8.5)$ & \\
\hline $\mathrm{BMI}, \mathrm{kg} / \mathrm{m}^{2}$, mean $\pm \mathrm{SD}$ & $26.0(4.7$ & $26.8(5.0$ & $28.1(5.4$ & $29.5(5.9$ & $<0.001$ \\
\hline Self-rated health, $n(\%)$ & & & & & $<0.001$ \\
\hline Excellent or very good & $776(64.4)$ & $658(54.5)$ & $646(53.6)$ & $553(45.9)$ & \\
\hline Good & $371(30.8)$ & $467(38.7)$ & $467(38.8)$ & $515(42.7)$ & \\
\hline Fair or poor & $58(4.8)$ & $82(6.8)$ & $92(7.6)$ & $138(11.4)$ & \\
\hline Physical function (SF-36), mean \pm SD & $81.0 \pm 19.7$ & $73.8 \pm 23.1$ & $69.2 \pm 24.5$ & $58.6 \pm 27.2$ & $<0.001$ \\
\hline Parental history of diabetes, $n(\%)$ & $397(32.9)$ & $392(32.6)$ & $415(34.4)$ & $395(32.8)$ & 0.76 \\
\hline History of hypertension, $n(\%)$ & $736(60.8)$ & $792(65.5)$ & 869 (71.9) & $913(75.5)$ & $<0.001$ \\
\hline No. of chronic conditionst, $n(\%)$ & & & & & $<0.001$ \\
\hline None & $187(15.5)$ & $152(12.6)$ & $115(9.5)$ & $94(7.8)$ & \\
\hline $1-2$ & $459(37.9)$ & $452(37.4)$ & $377(31.2)$ & $371(30.7)$ & \\
\hline$\geq 3$ & $414(34.2)$ & $395(32.6)$ & 479 (39.6) & $432(35.7)$ & \\
\hline $\mathrm{ST}, \mathrm{min} /$ day, mean $\pm \mathrm{SD} \ddagger$ & $432.9 \pm 48.4$ & $524.0 \pm 17.9$ & $584.5 \pm 17.2$ & $662.8 \pm 38.0$ & $<0.001$ \\
\hline Sedentary bout duration, min, mean $\pm S D$ & $5.1 \pm 0.9$ & $6.3 \pm 1.0$ & $7.6 \pm 1.3$ & $10.3 \pm 3.2$ & $<0.001$ \\
\hline MVPA, min/day, mean \pm SD $\ddagger$ & $84.9 \pm 37.6$ & $56.8 \pm 27.1$ & $42.0 \pm 21.9$ & $26.9 \pm 17.4$ & $<0.001$ \\
\hline Light $\mathrm{PA}, \mathrm{min} /$ day, mean $\pm \mathrm{SD} \ddagger$ & $377.0 \pm 47.8$ & $314.1 \pm 29.9$ & $268.3 \pm 25.3$ & $205.1 \pm 34.8$ & $<0.001$ \\
\hline
\end{tabular}

ST quartile cut points: Q1, 196-491 min; Q2, 492-554 min; Q3, 555-615 min; Q4, 616-830 min. $P$ value: $\chi^{2}$ for categorical variables and trend test for continuous. Boldface type indicates $P<0.05$. TChronic conditions: cardiovascular disease, cancer, cognitive impairment, depression, osteoarthritis, history of falls, chronic obstructive pulmonary disease, hypertension, cerebrovascular disease. $¥$ We adjusted for awake wear time using the residuals method.

When stratified analyses were further adjusted for MVPA + BMI, HRs for 1 IQR increment in ST were 1.04 $(95 \% \mathrm{Cl}$ 0.80-1.36) among women with $\mathrm{BMI}<30 \mathrm{~kg} / \mathrm{m}^{2}$ and $0.91(95 \%$ $\mathrm{Cl} 0.62-1.28)$ among women with higher BMI $\left(P_{\text {interaction }}=0.09\right.$ ) (Supplementary Table 2). And for family history, HRs were $0.90 \quad(95 \% \mathrm{Cl}$ 0.68-1.19) for women without a family history of diabetes and 1.15 (95\% Cl 0.81-1.64) among women with a family history; $P_{\text {interaction }}=0.15$.

Crude incidence rates for diabetes per 1,000 person-years in Q1, Q2, Q3, and Q4 of mean bout duration were 15.1, 15.7, 18.6, and 16.0, respectively (Table 2). Following adjustment for model 2 covariates, mean bout duration was not associated with risk for diabetes (Q4 vs. Q1 HR 1.01 [95\% Cl 0.72-1.42]; $P_{\text {trend }}=0.08$ ).

There was no statistical evidence of effect modification of mean bout duration in association with incident diabetes by age, BMI, physical functioning, MVPA, race/ethnicity, or family history of diabetes (Supplementary Tables 1 and 2).

\section{Isotemporal Analyses}

We performed isotemporal substitution analyses using model $3 a$ covariates and estimated the association with incident diabetes when statistically replacing $30 \mathrm{~min}$ per day of ST with $30 \mathrm{~min}$ of light PA or MVPA. The results, shown in Fig. 1, indicate that each $30-\mathrm{min}$ substitution reducing ST by adding light-PA time was associated with an HR of 0.99
(95\% Cl 0.94-1.04), whereas each 30-min substitution of ST with MVPA was associated with a $15 \%$ lower risk for diabetes (HR 0.85 [95\% Cl 0.75-0.96]). Isotemporal modeling indicated that statistically replacing 30 min of light PA with equal time in MVPA was also associated with $15 \%$ lower risk for incident diabetes (HR 0.85 [95\% Cl 0.73-0.98]; $P=0.03$ ).

\section{Sensitivity Analyses}

After removal from our analytic sample of 34 women (out of 342 total case subjects [9.9\%]) who reported newly diagnosed diabetes within the first 6 months of follow-up, ST HRs for Q1, Q2, Q3, and Q4 were 1.00 (reference), 1.11 (95\% Cl 0.80-1.54), $1.21(95 \% \mathrm{Cl}$ $0.87-1.68)$, and 1.14 (95\% Cl 0.80-1.62), 
Table 2-ST, mean sedentary bout duration, and incident diabetes in older women: the OPACH Study (2012-2018)

\begin{tabular}{cccccc}
\multicolumn{7}{c}{} & Q1 (low) & Q2 & Q3 & Q4 (high) & $P_{\text {trend }}{ }^{\dagger}$ \\
\hline $\begin{array}{c}\text { ST } \\
\text { Diabetes cases } \\
\text { [rate§] }\end{array}$ & $73[13.3]$ & $88[16.6]$ & $94[18.1]$ & $87[17.6]$ & \\
Model 1 & 1 (ref) & $1.27(0.93-1.74)$ & $1.41(1.04-1.93)$ & $1.43(1.04-1.97)$ & 0.003 \\
Model 2 & 1 (ref) & $1.20(0.87-1.65)$ & $1.33(0.97-1.82)$ & $1.21(0.86-1.70)$ & 0.04 \\
Model 3a & 1 (ref) & $1.21(0.87-1.68)$ & $1.30(0.93-1.80)$ & $1.14(0.79-1.64)$ & 0.12 \\
Model 3b & 1 (ref) & $1.04(0.74-1.45)$ & $1.07(0.75-1.52)$ & $0.92(0.62-1.36)$ & 0.76 \\
Model 3c & 1 (ref) & $1.04(0.74-1.47)$ & $1.04(0.72-1.50)$ & $0.85(0.56-1.29)$ & 0.90 \\
Mean bout duration & & & & & \\
Diabetes cases & & & & & \\
[rate§] & $84[15.1]$ & $83[15.7]$ & $96[18.6]$ & $79[16.0]$ & \\
Model 1 & 1 (ref) & $1.08(0.79-1.46)$ & $1.29(0.96-1.74)$ & $1.15(0.84-1.60)$ & 0.008 \\
Model 2 & 1 (ref) & $1.09(0.80-1.48)$ & $1.20(0.88-1.63)$ & $1.01(0.72-1.42)$ & 0.08 \\
Model 3a & 1 (ref) & $1.08(0.79-1.48)$ & $1.15(0.84-1.58)$ & $0.97(0.68-1.38)$ & 0.06 \\
Model 3b & 1 (ref) & $0.98(0.72-1.34)$ & $1.04(0.75-1.43)$ & $0.83(0.58-1.19)$ & 0.45 \\
Model 3c & 1 (ref) & $0.98(0.71-1.36)$ & $1.00(0.72-1.40)$ & $0.80(0.55-1.17)$ & 0.34
\end{tabular}

Data are HR $(95 \% \mathrm{Cl})$ unless otherwise indicated. ST quartile cut points: Q1, 196-491 min; Q2, 492$554 \mathrm{~min} ; \mathrm{Q} 3,555-615 \mathrm{~min}$; Q4, 616-830 $\mathrm{min}$. Mean sedentary bout duration quartile cut points: Q1, 2.6-5.5 min; Q2, 5.6-6.7 min; Q3, 6.8-8.3 min; Q4, 8.4-52.4 min. Model 1, adjustment for age and ethnicity $(n=4,839)$; model 2, model 1 adjustments + potential confounders $(n=4,734)$; model 3a, model 2 adjustments + BMI $(n=4,438)$; model $3 b$, model 2 adjustments + MVPA $(n=$ 4,734); model 3c, model 2 adjustments + BMI and MVPA $(n=4,438)$. Potential confounders include education, self-reported health, family history of diabetes, number of chronic conditions, physical functioning (SF-36), alcohol consumption, and current smoking status. Boldface type indicates $P<0.05$. ref, reference. $+P$ values from Cox multivariable linear regression models including ST in models in continuous form. $¥ W$ We adjusted for awake wear time using the residuals method. §Crude incidence rate per 1,000 person-years.

respectively; $P_{\text {trend }}=0.09$. Each $30-\mathrm{min}$ substitution reducing ST by adding light-PA time was associated with incident diabetes HR of 1.01 (95\% Cl 0.951.07), while adding MVPA was associated with incident diabetes $\mathrm{HR}$ of $0.84(95 \% \mathrm{Cl}$ 0.74-0.96). After removal of 147 women with fasting glucose $\geq 126 \mathrm{mg} / \mathrm{dL}$ from a subsample $(n=3,832)$ who had phlebotomy, ST HRs for Q1, Q2, Q3, and Q4 were 1.00 (reference), 1.18 ( $95 \% \mathrm{Cl} 0.81$ 1.71), 1.21 (95\% Cl 0.83-1.76), and 1.08 $(95 \% \mathrm{Cl} 0.72-1.63) ; P_{\text {trend }}=0.28$. Each 30-min substitution reducing ST by adding light PA time was associated with incident diabetes HR of $1.03(95 \% \mathrm{Cl}$ 0.97-1.10), while adding MVPA was associated with incident diabetes HR of 0.82 (95\% Cl 0.71-0.95). In the full subsample ( $n=3,832)$, isotemporal substitution results were nearly identical to those above after additional adjustment for fasting glucose and insulin. There were no changes when the consistent sample was used.

\section{CONCLUSIONS}

In this cohort of older ambulatory women, nearly half of whom were over age 80 years, higher accelerometer-measured ST was associated with greater risk for diabetes, though the association was not independent of BMI or MVPA. Use of isotemporal substitution analysis to hypothetically replace $30 \mathrm{~min} /$ day of ST with equivalent time spent in MVPA resulted in a 15\% lower risk for diabetes. Replacing $30 \mathrm{~min}$ of light PA with MVPA was also associated with a $15 \%$ lower risk for diabetes, whereas replacement of ST with light PA was not associated with lower risk for diabetes. Study results failed to confirm our hypothesis, based on acute and cross-sectional diabetes studies and longitudinal studies that used self-reported ST, that longer STs would be associated with higher risk for diabetes in late life, and suggest that moderate reductions in ST may be insufficient to delay or prevent diabetes unless MVPA is increased. We also did not observe significant associations between ST patterns and incident diabetes.

Epidemiologic studies, mostly in younger and middle-aged adults, have shown strong and consistent associations between self-reported TV time and incident diabetes, even after adjustment for MVPA (8). However, TV time might reflect an interaction of several health behaviors (sitting + snacking, for example) or lifestyle patterns that have independent effects on incident diabetes (25). When sitting time (a measure that includes other sedentary behaviors in addition to TV time specifically) has been assessed by self-report, associations with incident diabetes have been mixed, with some studies reporting associations only among those with high $\mathrm{BMI}$ and/or low MVPA and one showing null results with adjustment for BMI (26-29). For example, among 88,829 participants of the WHI observational study (mean \pm SD age $=62 \pm 7$ years) followed for an average of 14.4 years, obese women who self-reported sitting $\geq 16 \mathrm{~h}$ per day had 1.25 times higher odds of new-onset diabetes than obese women reporting sitting $\leq 7 \mathrm{~h}$ per day (29); however, no significant associations were observed for normal weight or overweight women. Our contrasting results from the current study that associations of ST are higher in women with $<30 \mathrm{~kg} / \mathrm{m}^{2}$ BMI suggest possibly differential mechanisms for ST and incident diabetes associations that depend on age of diabetes onset-for example, among women with diabetes onset at a younger age, fat accretion associated with ST (30) might be a mechanism, while among women with older-onset diabetes, accelerated sarcopenia associated with ST (31) might play a role. Future studies over longer periods of time that include repeated measures for physical behavior, adiposity, and diabetes are now needed.

In our previous study within this OPACH Study cohort, we found strong associations of accelerometer-measured ST and ST patterns with prevalent diabetes at OPACH Study baseline (13). These findings remained strong after adjustment for $\mathrm{BMI}$ and MVPA and were present in every subgroup of women analyzed: high versus low MVPA, high versus low physical functioning, $\mathrm{BMI}>$ vs. $<30 \mathrm{~kg} / \mathrm{m}^{2}$; age $>$ vs. $<80$ years, family history of diabetes yes versus no, and race/ethnicity White, Black, Hispanic (13). In the current study on diabetes incidence, after removing from the OPACH Study cohort 1,282 women (20.9\% of the sample) who were diagnosed with diabetes earlier in their life, we focused exclusively on incident diabetes cases in our older adult sample. Newly diagnosed diabetes among older adults, compared with younger adults, is more likely to be asymptomatic (32) and milder, and therefore abnormal glucose levels may be less likely to be labeled as diabetes in late life. Also, the pathophysiology of newly 


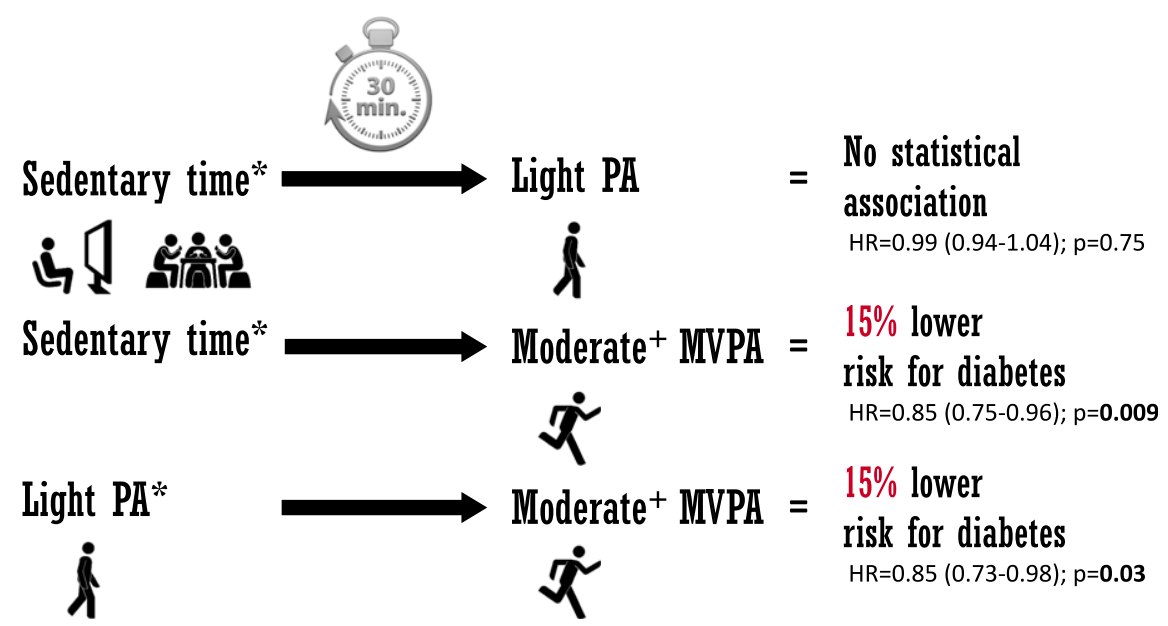

Figure 1-Statistically replacing (reducing) ST with (increasing) light PA or MVPA and statistically replacing (reducing) light PA with (increasing) MVPA. Results from isotemporal substitution Cox proportional hazards models adjusted for age, race/ethnicity, education, smoking status, alcohol use, family history of diabetes, multimorbidity, physical functioning, self-rated health, and BMI. HRs are shown with $95 \%$ Cls. *Statistically replacing 30 min with light PA or MVPA.

acquired diabetes in older adults is different than in younger adults; adults over 75 years of age have age-related changes in mitochondrial dysfunction and sarcopenia that can accelerate decreased insulin secretion and increased insulin resistance, leading to clinical diabetes at older ages (33). The accelerated deficiencies in glucose metabolism may require a larger neurohormonal and metabolic counterregulatory stimulus to mitigate progression to frank diabetes. This could explain why we did not observe a clear inverse association with diabetes incidence when hypothetically replacing sitting time with equivalent time spent in light-intensity PA, whereas we did find a beneficial association for replacement with MVPA. A recent compositional analysis of accelerometermeasured MVPA, light PA, and ST with biomarkers of prediabetes and type 2 diabetes in an adult sample, mean \pm SD age $=64.4 \pm 7.7$ years, lends support to this speculation. Investigators found that statistically substituting light PA for ST had generally small associations, while substituting MVPA for ST was related to larger, beneficial associations with most biomarkers (34). Indeed, controlled trials among older men and women have shown that higher-intensity activities like combining resistance training and aerobic exercise can maximize weight loss, skeletal muscle mass gain, and strength gain and can improve insulin resistance (5). The current study shows that MVPA, when replacing ST, is associated with lower diabetes risk.
These results are supported by experimental studies showing that engaging in lifestyle modification that is heavily focused on improving diet and increasing MVPA can prevent or delay the onset of diabetes (35), even 10 years following intervention, with more pronounced effects in older adults than younger adults (36).

In the only other prospective study of accelerometer-measured ST and incident diabetes that we found, over a 5 -year period, 81 cases of incident diabetes were reported among 1,718 U.S. adults mean \pm SD age $=45 \pm 3$ years, and the odds of incident diabetes did not significantly vary by ST, with or without adjustment for MVPA (11). While our results suggested that ST was related to incident diabetes in older adults, as in our previous study, results were not statistically independent of MVPA. A common explanation for this lack of statistical independence is confounding, which in our case requires that 1) women with low ST also had high MVPA (the correlation between MVPA and ST in the OPACH Study was -0.67), 2) MVPA is causally related to incident diabetes, and 3) once MVPA is accounted for, associations between ST and diabetes are attenuated-overall, the confounding explanation implies that the initial ST and diabetes association was at least somewhat spurious. Another explanation for the lack of association when MVPA was accounted for is that the behaviors themselves are interdependent, such that reducing one will cause increases in another, which is the case with ST and PA. The results of our isotemporal substitution analyses showing associations with incident diabetes after statistically reducing ST and replacing it with an equal amount of MVPA time suggest that this latter explanation is plausible. Furthermore, the null results observed for statistically replacing ST with light PA suggest that higher-intensity activity might be needed to prevent diabetes among older adults. This hypothesis was further supported by our findings that statistically replacing light PA with equal time in MVPA was associated with lower diabetes risk. Future studies that experimentally test these theories are needed, as are studies that investigate how patterns of sitting might play a role in metabolic dysregulation and late-life-onset diabetes in older adults.

\section{Limitations}

This study was limited by having shorter follow-up time than previous studies of reported sedentary behavior and incident diabetes. Despite the short followup, we had sufficient power to detect associations using multivariable Cox models, likely due to our use of objectively measured sedentary behavior and our relatively large sample size. The selfreported diabetes question used to identify diabetes did not distinguish between type 1 and type 2 diabetes; however, type 2 diabetes accounts for $>95 \%$ of total diagnosed diabetes in U.S. 
older adults (37) and the measure has demonstrated high accuracy when compared with physician adjudication based on medical record reviews (17). Still, use of reported medically treated diabetes may miss those with subclinical or undiagnosed disease, which among older adults occurs in $2.9 \%$ of the U.S. population (1). Assuming those with undiagnosed diabetes are more sedentary and engage in less MVPA, this could introduce a bias toward the null, suggesting that our findings may underestimate the association between physical behavior and incident diabetes in older women. The detection of ST patterns in the current study was limited by measurement with hip-worn accelerometers, which are less accurate for identifying postural transitions than devices specifically designed for this purpose (e.g., activPAL) (38). Such measurement error may lead to misclassification that reduces effect sizes and could account for the null findings. Future studies would benefit from use of posture-based devices to evaluate ST patterns in relation to incident diabetes. Additionally, accelerometer data were collected over a period of $\leq 7$ days, which is adequately reproducible over a 2-3 year period (39) but may not reflect longer-term patterns of sedentary behavior. The current study was also conducted among older women only, and generalizability would be enhanced by testing of these associations in cohorts of older and younger women and men. Finally, the isotemporal substitution analysis relied on longitudinal data collected during an observational study in which there was no experimental intervention for replacement of behaviors. The results should therefore be interpreted as associations, and future studies are needed in older people to test whether interventions to replace ST with PA prevent incidence of type 2 diabetes in later life.

\section{Strengths}

Notable strengths of our study are the relatively large and diverse $(53.1 \%$ White, $30.4 \%$ Black, and $16.6 \%$ Hispanic) cohort of older community-dwelling women with objective measures of sedentary behavior based on a 24-h wear protocol. Objective measures are important for characterizing sedentary behavior because sitting is the default position for many older adults, making it an automatic behavior that is difficult to quantify by self-report (9). The need for higherquality evidence concerning diabetes in this older age-group was a major theme of a recent consensus statement by the American Diabetes Association (40). And the wide variation in race/ethnicity and educational attainment ( $42 \%$ had a bachelor's degree or higher) limited threats to external validity to older U.S. women. Finally, the prospective design of the study and sensitivity analyses are also distinct strengths in that they help establish temporal order of the association between sedentary behavior and diabetes.

In conclusion, our findings indicate that reducing ST or light PA while increasing MVPA might mitigate diabetes risk in older women. Findings also suggest that substituting time in light PA for ST may be insufficient to delay or prevent diabetes in late life; replacement of ST by the stronger physiologic stimulus provided by MVPA may be necessary. Because older adults have a lower resting metabolic rate and higher energy cost of movement, the types of PA and efforts needed to achieve MVPA are different for them than for younger adults. For example, a usual-paced 400-m walk was shown to be sufficient to achieve MVPA for most women average age 80 years (18). MVPA has also been shown to be attainable for ambulatory older adults even in the presence of clinical mobility limitations (41). Our results, which pertain to older ambulatory community-living women who reach late life without getting diabetes, add to mounting evidence warranting the conduct of interventions in age-, sex-, and ethnicitydiverse populations to test replacement of ST with MVPA for the prevention of late-lifeonset diabetes. Until data from such trials are available, the totality of the evidence related to cardiometabolic health supports recommendations, such as the national 2018 Physical Activity Guidelines for Americans and those from the American Diabetes Association (42), to reduce ST in whatever ways are feasible, safe, and easy to incorporate into daily life and to increase PA levels as part of a healthy lifestyle strategy aimed at improvement of personal and public health.

Acknowledgments. The full list of WHI investigators responsible for the overarching project in which the authors' study was nested can be found at the following website: https://www.whi .org/researchers/Documents\%20\%20Write\%20a\% 20Paper/WHI\%2OInvestigator\%20Long\%20List.pdf.
Funding. The National Heart, Lung, and Blood Institute (NHLBI), National Institutes of Health, provided funding for the OPACH Study (grant R01 HL105065 to A.Z.L.). Funding also came from training grants provided by NHLBI (T32 HL079891 to J.B.) and a program project (P01 AG052352 to A.Z.L.) supported by the National Institute on Aging. The WHI program was funded by the NHLBI, National Institutes of Health, U.S. Department of Health and Human Services (HHSN268201600018C, HHSN268201600001C, HHSN268201600002C, HHSN268201600003C, and HHSN268201600004C). G.N.H. was supported by an Australian National Health and Medical Research Council Career Development Fellowship (no. 108029).

Duality of Interest. No potential conflicts of interest relevant to this article were reported. Author Contributions. J.B., A.Z.L., and M.J.L. contributed to the study concept and design. J.B., A.Z.L., G.N.H., M.J.L., C.D., K.R.E., I-M.L., M.F.H., and J.K. contributed to acquisition, analysis, or interpretation of data. J.B., A.Z.L., and S.L. contributed to drafting of the manuscript. J.B., A.Z.L., G.N.H., M.J.L., C.D., K.R.E., I-M.L., M.F.H., J.K., E.R.-S., and D.B. contributed to critical revision of the manuscript for important intellectual content. J.B. contributed to statistical analysis. A.Z.L., K.R.E., D.B., and M.J.L. obtained funding. E.R.-S. and D.B. provided administrative, technical, or material support. J.B. is the guarantor of this work and, as such, had full access to all the data in the study and takes responsibility for the integrity of the data and the accuracy of the data analysis.

Prior Presentation. Parts of this study were presented in abstract form at the Gerontological Society of America 2018 Annual Scientific Meeting, Boston, MA, 14-18 November 2018.

\section{References}

1. Centers for Disease Control and Prevention. National Diabetes Statistics Report, 2020. Atlanta, GA, Centers for Disease Control and Prevention, 2020

2. Meneilly GS, Tessier D. Diabetes in elderly adults. J Gerontol A Biol Sci Med Sci 2001;56: M5-M13

3. Booth GL, Kapral MK, Fung K, Tu JV. Relation between age and cardiovascular disease in men and women with diabetes compared with nondiabetic people: a population-based retrospective cohort study. Lancet 2006;368:29-36

4. Atkins RC. The epidemiology of chronic kidney disease. Kidney Int Suppl 2005;67:S14-S18

5. Kalyani RR, Corriere M, Ferrucci L. Age-related and disease-related muscle loss: the effect of diabetes, obesity, and other diseases. Lancet Diabetes Endocrinol 2014;2:819-829

6. Mozaffarian D, Kamineni A, Carnethon M, Djoussé L, Mukamal KJ, Siscovick D. Lifestyle risk factors and new-onset diabetes mellitus in older adults: the cardiovascular health study. Arch Intern Med 2009;169:798-807

7. Tremblay MS, Aubert S, Barnes JD, et al.; SBRN Terminology Consensus Project Participants. Sedentary Behavior Research Network (SBRN)

- Terminology Consensus Project process and outcome. Int J Behav Nutr Phys Act 2017;14: 75

8. Biswas A, Oh PI, Faulkner GE, et al. Sedentary time and its association with risk for disease incidence, mortality, and hospitalization in adults: a systematic review and meta-analysis 
[published correction appears in Ann Intern Med 2015;163:400]. Ann Intern Med 2015;162:123-132 9. LaMonte MJ, Lee I-M, Rillamas-Sun E, et al. Comparison of questionnaire and device measures of physical activity and sedentary behavior in a multi-ethnic cohort of older women. J Meas Phys Behav 2019;2:82-93

10. Brocklebank LA, Falconer CL, Page AS, Perry $R$, Cooper AR. Accelerometer-measured sedentary time and cardiometabolic biomarkers: a systematic review. Prev Med 2015;76:92-102

11. Barone Gibbs B, Pettee Gabriel K, Reis JP, Jakicic JM, Carnethon MR, Sternfeld B. Crosssectional and longitudinal associations between objectively measured sedentary time and metabolic disease: the Coronary Artery Risk Development in Young Adults (CARDIA) study. Diabetes Care 2015;38:1835-1843

12. van der Berg JD, Stehouwer CDA, Bosma $\mathrm{H}_{\text {, }}$ et al. Associations of total amount and patterns of sedentary behaviour with type 2 diabetes and the metabolic syndrome: the Maastricht Study. Diabetologia 2016;59:709-718

13. Bellettiere J, Healy GN, LaMonte MJ, et al. Sedentary behavior and prevalent diabetes in 6,166 older women: the Objective Physical Activity and Cardiovascular Health Study. J Gerontol A Biol Sci Med Sci 2019;74:387-395

14. Gibbs BB, Hergenroeder AL, Katzmarzyk PT, Lee IM, Jakicic JM. Definition, measurement, and health risks associated with sedentary behavior. Med Sci Sports Exerc 2015;47:1295-1300

15. LaCroix AZ, Rillamas-Sun E, Buchner D, et al. The Objective Physical Activity and Cardiovascular Disease Health in Older Women (OPACH) Study. BMC Public Health 2017;17:192

16. Migueles JH, Cadenas-Sanchez C, Ekelund U, et al. Accelerometer data collection and processing criteria to assess physical activity and other outcomes: a systematic review and practical considerations. Sports Med 2017;47:1821-1845 17. Jackson JM, DeFor TA, Crain AL, et al. Validity of diabetes self-reports in the Women's Health Initiative. Menopause 2014;21:861-868

18. Evenson KR, Wen F, Herring AH, et al. Calibrating physical activity intensity for hip-worn accelerometry in women age 60 to 91 years: the Women's Health Initiative OPACH Calibration Study. Prev Med Rep 2015;2:750-756

19. Shiroma EJ, Freedson PS, Trost SG, Lee I-M. Patterns of accelerometer-assessed sedentary behavior in older women. JAMA 2013;310:25622563
20. Ware JE Jr., Sherbourne CD. The MOS 36-item short-form health survey (SF-36). I. Conceptual framework and item selection. Med Care 1992;30:473-483

21. Pedišić Ž. Measurement issues and poor adjustments for physical activity and sleep undermine sedentary behaviour research - the focus should shift to the balance between sleep, sedentary behavior, standing and activity. Kinesiol Int J Fundam Appl Kinesiol 2014;46:135146

22. Chastin SFM, Palarea-Albaladejo J, Dontje ML, Skelton DA. Combined effects of time spent in physical activity, sedentary behaviors and sleep on obesity and cardio-metabolic health markers: a novel compositional data analysis approach. PLoS One 2015;10:e0139984

23. Page A, Peeters G, Merom D. Adjustment for physical activity in studies of sedentary behaviour. Emerg Themes Epidemiol 2015;12:10

24. Mekary RA, Willett WC, Hu FB, Ding EL. Isotemporal substitution paradigm for physical activity epidemiology and weight change. Am J Epidemiol 2009;170:519-527

25. Hamer M, Ding D, Chau J, Duncan MJ, Stamatakis E. Association between TV viewing and heart disease mortality: observational study using negative control outcome. J Epidemiol Community Health 2020;74:391-394

26. Åsvold BO, Midthjell K, Krokstad S, Rangul V, Bauman A. Prolonged sitting may increase diabetes risk in physically inactive individuals: an 11 year follow-up of the HUNT Study, Norway. Diabetologia 2017;60:830-835

27. Petersen CB, Bauman A, Tolstrup JS. Total sitting time and the risk of incident diabetes in Danish adults (the DANHES cohort) over 5 years: a prospective study. Br J Sports Med 2016;50: 1382-1387

28. Stamatakis E, Pulsford RM, Brunner EJ, et al. Sitting behaviour is not associated with incident diabetes over 13 years: the Whitehall II cohort study. Br J Sports Med 2017;51:818-823

29. Manini TM, Lamonte MJ, Seguin RA, et al. Modifying effect of obesity on the association between sitting and incident diabetes in postmenopausal women. Obesity (Silver Spring) 2014; 22:1133-1141

30. Hu FB, Li TY, Colditz GA, Willett WC, Manson JE. Television watching and other sedentary behaviors in relation to risk of obesity and type 2 diabetes mellitus in women. JAMA 2003;289: 1785-1791
31. Kortebein P, Symons TB, Ferrando A, et al. Functional impact of 10 days of bed rest in healthy older adults. J Gerontol A Biol Sci Med Sci 2008;63:1076-1081

32. Sinclair A, Dunning T, Rodriguez-Mañas L. Diabetes in older people: new insights and remaining challenges. Lancet Diabetes Endocrinol 2014;3:275-285

33. Meneilly GS. Pathophysiology of diabetes in the elderly. In Diabetes in Old Age. 3rd ed. Sinclair AJ, Ed. Chichester, West Sussex, U.K. John Wiley \& Sons, Ltd, 2009

34. Rossen J, Von Rosen P, Johansson U-B, Brismar K, Hagströmer M. Associations of physical activity and sedentary behavior with cardiometabolic biomarkers in prediabetes and type 2 diabetes: a compositional data analysis. Phys Sportsmed 2020;48:222-228

35. Knowler WC, Barrett-Connor E, Fowler SE, et al.; Diabetes Prevention Program Research Group. Reduction in the incidence of type 2 diabetes with lifestyle intervention or metformin. N Engl J Med2002;346:393-403

36. Crandall J, Schade D, Ma Y, et al.; Diabetes Prevention Program Research Group. The influence of age on the effects of lifestyle modification and metformin in prevention of diabetes. $J$ Gerontol A Biol Sci Med Sci 2006;61:1075-1081 37. Bullard KM, Cowie CC, Lessem SE, et al. Prevalence of diagnosed diabetes in adults by diabetes type - United States, 2016. MMWR Morb Mortal Wkly Rep 2018;67:359-361

38. Lyden K, Kozey Keadle SL, Staudenmayer JW, Freedson PS. Validity of two wearable monitors to estimate breaks from sedentary time. Med Sci Sports Exerc 2012;44:2243-2252

39. Keadle SK, Shiroma EJ, Kamada M, Matthews CE, Harris TB, Lee I-M. Reproducibility of accelerometerassessed physical activity and sedentary time. Am J Prev Med 2017;52:541-548

40. Kirkman MS, Briscoe VJ, Clark N, et al.; Consensus Development Conference on Diabetes and Older Adults. Diabetes in older adults: a consensus report. J Am Geriatr Soc 2012;60: 2342-2356

41. Bellettiere J, Lamonte MJ, Unkart J, et al. Short physical performance battery and incident cardiovascular events among older women. J Am Heart Assoc 2020;9:e016845

42. Colberg SR, Sigal RJ, Yardley JE, et al. Physical activity/exercise and diabetes: a position statement of the American Diabetes Association. Diabetes Care 2016;39:2065-2079 